\title{
Avaliação comparativa da altura patelar pós-artroplastias totais do joelho com e sem preservação do ligamento cruzado posterior*
}

\author{
Comparative evaluation of patellar height \\ after total knee arthroplasty with and without \\ preservation of the posterior cruciate ligament
}

\author{
Alfredo Maroues Villardi ${ }^{1}$, JoÃo Paulo Bezerra Leite ${ }^{2}$, \\ BRUNo Figueiredo dos SANTOS ${ }^{3}$, MARCELO MANDARINO ${ }^{4}$
}

\section{RESUMO}

Objetivo: Avaliar possíveis alterações na altura da patela, pós-artroplastias totais do joelho com e sem preservação do ligamento cruzado posterior (LCP). Método: Foram analisadas radiografias pré e pósoperatórias, de 120 pacientes submetidos a artroplastias totais do joelho, realizadas no Instituto Nacional de Traumatologia e Ortopedia, no período compreendido entre janeiro de 2003 e janeiro de 2005, com implantes do tipo Johnson de plataforma rotatória. A amostra foi dividida em dois

* Trabalho realizado no Instituto Nacional de Traumatologia e Ortopedia - INTO - Rio de Janeiro (RJ), Brasil.

1. Pós-graduando do Programa de Pós-Graduação de Ciências Médicas da UERJ, Mestre em Medicina pela Faculdade de Medicina da UFRJ, Membro do Grupo de Joelho do Instituto Nacional de Traumatologia e Ortopedia - INTO - Rio de Janeiro (RJ), Brasil.

2. Residente do 3o ano do Programa de Residência Médica do Instituto Nacional de Traumatologia e Ortopedia - INTO - Rio de Janeiro (RJ), Brasil.

3. Médico Ortopedista, Membro do Serviço de Ortopedia do Hospital Geral de Roraima (RO), Brasil.

4. Médico Ortopedista, Membro do Serviço de Ortopedia e Traumatologia do Hospital São Vicente de Paulo - Rio de Janeiro (RJ), Brasil.

Endereço para correspondência: Av. Maracanã, 3.200/C01, Tijuca -20530-231 - Rio de Janeiro, RJ. Tels.: (21) 2278-9177/9112-8511.

E-mail: avillardi@ig.com.br

Recebido em 27/11/07. Aprovado para publicação em 6/5/08.

Copyright RBO2008 grupos, correspondendo o grupo I a exames radiográficos pré e pós-artroplastias totais do joelho com preservação do LCP $(n=60)$ e o grupo II, a exames radiográficos pré e pós-artroplastias sem preservação do LCP $(n=60)$. Para a mensuração dos valores pré e pós-operatórios foi utilizado o método de Blackburne-Peel, cujo valor normal é igual a 0,8 $( \pm 0,2)$, variando, portanto, entre 0,6 e 1 . Resultados: $O$ índice patelar médio pré-operatório do grupo I foi igual a 0,874 e, no grupo II, a média foi igual a 0,857 . $O$ índice médio de altura patelar pósoperatório do grupo I foi igual a $\mathbf{0 , 8 4 9}$, variando entre 0,54 e 1,03. No grupo II, o menor índice foi igual a 0,48 , o maior igual a 0,94 , sendo o índice médio igual a 0,79 . A comparação dos valores pósoperatórios nos dois grupos apresentou diferença estatisticamente significativa $(\mathbf{p}<0,05)$. Conclusões: Foi observada tendência à diminuição da altura patelar em ambos os grupos estudados, sendo esta mais evidente no grupo em que houve sacrifício do LCP.

Descritores - Ligamento cruzado posterior; Patela/radiografia; Artroplastia do joelho/métodos

\section{ABSTRACT}

Objective: To evaluate possible changes in patellar height after total knee arthroplasties with and without preservation of the posterior cruciate ligament (PCL). Method: Analysis was made of pre-and post-operative 
$X$-rays of 120 patients submitted to total knee arthroplasties at the National Institute of Traumatology and Orthopedics between January 2003 and January 2005, with Johnson-type rotary platform implants. The sample was divided into two groups, group I corresponding to radiographic exams before and after total knee arthroplasty with preservation of the PCL $(n=$ 60), and group II corresponding to radiographic exams before and after arthroplasties without preservation of the PCL $(n=60)$. To measure the values before and after surgery, the authors used the Blackburne - Peel method, whose normal value is equal to 0.8 ( \pm 0.2 ), therefore varying between 0.6 and 1. Results: The mean preoperative patellar ratio in group I was equal to 0.874 , and in group II the mean was equal to 0.857. The mean post-operative patellar height ratio in group I was equal to 0.849 , varying between 0.54 and 1.03. In group II, the lower ratio was equal to 0.48 , and the highest, equal to 0.94, the mean rate being equal to 0.79. The comparison of post-operative value between the groups presented a statistically significant difference $(p<0.05)$. Conclusions: A tendency towards decreased patellar height was seen in both groups studied, but it was more evident in the group in which the PCL was not preserved.

Keywords - Posterior cruciate ligament; Patella/radiography; Arthroplasty, replacement, knee/methods

\section{INTRODUÇÃO}

A patela exerce papel importante na biomecânica do joelho, alongando o braço de alavanca do mecanismo extensor, portanto, aumentando a força do quadríceps entre 30 e 50\%. A articulação patelofemoral cria uma força de reação articular que se relaciona tanto ao grau de flexão em que se encontra o joelho, quanto à contração quadricipital ${ }^{(1-2)}$.

Tanto a patela alta quanto a baixa podem produzir alterações estruturais do ligamento patelar, do tendão quadricipital e instabilidade patelofemoral ${ }^{(3-5)}$. Queixas patelofemorais são responsáveis por grande percentual de revisões de artroplastias totais de joelho ${ }^{(6-10)}$.

Uma das grandes controvérsias no capítulo das artroplastias totais do joelho é a preservação ou não do ligamento cruzado posterior (LCP). Na literatura, não há consenso quanto ao melhor procedimento a ser adotado, em relação ao LCP, principalmente, em relação aos portadores de osteoartrose do joelho ${ }^{(11-12)}$.

A altura da patela pós-artroplastia total de joelho tem especial interesse, principalmente pela sua vinculação com a interlinha articular e guarda relação com o prévio posicionamento da patela e com ações intraoperatórias, como, por exemplo, a decisão de se preservar ou não o LCP.

Conforme demonstrado na literatura, a altura patelar tem importante influência na cinemática do joelho, sendo o seu posicionamento pós-artroplastia total determinante da função articular e da longevidade do implante $^{(13-15)}$.

A partir da avaliação dos resultados obtidos, os comparamos com aqueles descritos na literatura ortopédica, com o propósito de avaliar se a preservação do LCP na artroplastia total do joelho exerce ou não influência sobre a altura pós-operatória da patela. Dessa forma, esperamos obter mais elementos que, associados a outros, possam nos auxiliar a definir qual a melhor opção em relação à preservação ou não do LCP nas artroplastias totais do joelho.

O objetivo deste trabalho foi avaliar radiograficamente a altura patelar pré e pós-artroplastia total do joelho, pelo método de Blackburne-Peel ${ }^{(16)}$, comparando os resultados nos grupos em que se preservou o LCP com aqueles outros nos quais o ligamento foi ressecado.

\section{MÉTODOS}

No período entre janeiro de 2003 e janeiro de 2005, foram realizadas 431 ATJ no Instituto Nacional de Traumatologia e Ortopedia, com implantes $P F C^{\circledR}$ de plataforma rotatória (Johnson \& Johnson ${ }^{\circledR}$, Warshaw - EUA). Desse universo, foram escolhidas aleatoriamente, por sorteio, 140 ATJ, com e sem preservação do LCP, a fim de avaliar, com o índice de Blackburne-Peel ${ }^{(16)}$, possíveis repercussões na altura da patela, devido à preservação ou não do LCP.

O número amostral por nós estabelecido no desenho da pesquisa corresponde a $32,48 \%$ do universo de artroplastias de joelho realizadas no período descrito, 
percentual estatisticamente expressivo como estimativa populacional.

Das 140 radiografias sorteadas, foram excluídos 20 casos, por apresentar patela baixa ou alta pré-operatória (fora do intervalo de 0,6 a 1) ou aqueles em que as radiografias não obedeceram à padronização de incidência e/ou angulação sagital exigidas, a fim de homogeneizar a amostra e evitar possíveis vieses. Portanto, foram avaliados 120 pares de radiografias, pré e pós-artroplastias totais do joelho, divididos em dois grupos, de acordo com a preservação ou não do LCP, sendo alocados 60 pares de radiografias em cada. O grupo I foi constituído por radiografias pré e pós-ar-

TABELA 1

Índices patelares do grupo I, com preservação do LCP

\begin{tabular}{|c|c|c|c|c|c|}
\hline ID & Pré & Pós & ID & Pré & Pós \\
\hline 14074 & 0,9 & 0,78 & 103360 & 0,88 & 0,9 \\
\hline 33777 & 1 & 1,03 & 103566 & 0,83 & 0,86 \\
\hline 50286 & 0,7 & 0,62 & 105857 & 0,88 & 0,96 \\
\hline 53980 & 0,96 & 0,9 & 106237 & 0,6 & 0,64 \\
\hline 66771 & 0,92 & 0,86 & 106712 & 0,87 & 0,82 \\
\hline 74229 & 0,88 & 0,86 & 107570 & 0,92 & 0,95 \\
\hline 81264 & 0,62 & 0,56 & 107804 & 0,9 & 0,87 \\
\hline 81536 & 0,8 & 0,77 & 109832 & 0,84 & 0,93 \\
\hline 82634 & 1 & 0,96 & 109863 & 0,87 & 0,8 \\
\hline 86822 & 0,6 & 0,54 & 109868 & 0,91 & 0,89 \\
\hline 87879 & 0,78 & 0,8 & 109931 & 0,78 & 0,74 \\
\hline 90596 & 0,9 & 0,92 & 110248 & 0,88 & 0,85 \\
\hline 90888 & 0,88 & 0,88 & 110317 & 0,82 & 0,82 \\
\hline 91743 & 1 & 0,87 & 110402 & 0,86 & 0,83 \\
\hline 92087 & 0,88 & 0,87 & 110487 & 1 & 0,89 \\
\hline 93643 & 0,83 & 0,75 & 110953 & 0,93 & 0,9 \\
\hline 93875 & 0,68 & 0,58 & 111160 & 1 & 0,89 \\
\hline 99300 & 0,84 & 0,88 & 112881 & 0,96 & 0,9 \\
\hline 99680 & 0,84 & 0,88 & 112914 & 0,9 & 0,96 \\
\hline 99778 & 0,8 & 0,82 & 113172 & 0,98 & 0,96 \\
\hline 100339 & 0,87 & 0,81 & 113774 & 0,8 & 0,78 \\
\hline 100498 & 0,96 & 0,93 & 114764 & 0,91 & 0,86 \\
\hline 100521 & 0,96 & 0,96 & 115603 & 0,83 & 0,86 \\
\hline 101052 & 0,98 & 0,87 & 115772 & 0,83 & 0,81 \\
\hline 101224 & 0,84 & 0,96 & 115910 & 0,84 & 0,82 \\
\hline 101363 & 1 & 0,9 & 117737 & 0,92 & 0,88 \\
\hline 101641 & 0,96 & 0,93 & 118606 & 0,96 & 0,88 \\
\hline 102716 & 0,68 & 0,62 & 121057 & 0,98 & 0,92 \\
\hline 103110 & 0,9 & 0,92 & 121192 & 0,96 & 0,96 \\
\hline 103251 & 0,96 & 0,94 & 121759 & 0,92 & 0,86 \\
\hline
\end{tabular}

Legenda: ID: Número do prontuário; Pré: Índices pré-operatórios; Pós: Índices pósoperatórios.

Fonte: INTO/MS 2003-2007. troplastias com preservação do LCP, enquanto no grupo II foram incluídas as radiografias de artroplastias sem preservação do LCP (tabelas 1 e 2).

A altura patelar pré e pós-operatória foi mensurada nas radiografias do joelho, em $30^{\circ}$ de flexão, na incidência lateral, pelo método de Blackburne-Peel ${ }^{(16)}$. Esse índice é expresso pela razão entre a distância de uma linha traçada sobre o planalto tibial, até a borda inferior da superfície articular da patela (A), e o comprimento da superfície articular da patela (B), ou seja, $\mathrm{IP}_{\mathrm{BP}}=\mathrm{A} / \mathrm{B}$. O valor normal desse índice é igual a 0,8 ( \pm $0,2)$, variando, portanto, a faixa de normalidade entre 0,6 e $1,0^{(16)}$ (figura 1 ).

\section{TABELA 2}

Índices patelares do grupo II, sem preservação do LCP

\begin{tabular}{|c|c|c|c|c|c|}
\hline ID & Pré & Pós & ID & Pré & Pós \\
\hline 46132 & 0,78 & 0,7 & 124729 & 0,86 & 0,82 \\
\hline 65689 & 0,81 & 0,76 & 125212 & 0,93 & 0,84 \\
\hline 80521 & 0,94 & 0,88 & 125744 & 0,94 & 0,88 \\
\hline 88383 & 0,82 & 0,83 & 126888 & 0,8 & 0,76 \\
\hline 89986 & 0,68 & 0,58 & 127068 & 0,88 & 0,76 \\
\hline 95176 & 0,84 & 0,86 & 130932 & 0,9 & 0,82 \\
\hline 96351 & 1 & 0,81 & 131270 & 0,9 & 0,92 \\
\hline 97535 & 0,8 & 0,72 & 132411 & 0,96 & 0,9 \\
\hline 100550 & 0,86 & 0,8 & 133002 & 0,88 & 0,8 \\
\hline 105429 & 0,74 & 0,58 & 133605 & 0,86 & 0,94 \\
\hline 107746 & 0,8 & 0,62 & 133937 & 0,72 & 0,76 \\
\hline 107795 & 0,76 & 0,68 & 134115 & 1 & 0,9 \\
\hline 108215 & 0,83 & 0,72 & 134410 & 0,84 & 0,78 \\
\hline 108362 & 0,72 & 0,66 & 134498 & 0,68 & 0,55 \\
\hline 108455 & 0,78 & 0,73 & 135014 & 0,82 & 0,78 \\
\hline 108874 & 0,84 & 0,77 & 135395 & 0,88 & 0,8 \\
\hline 108879 & 0,93 & 0,81 & 135446 & 0,9 & 0,94 \\
\hline 108975 & 0,93 & 0,82 & 135858 & 0,62 & 0,48 \\
\hline 110427 & 0,9 & 0,79 & 136068 & 0,88 & 0,82 \\
\hline 113288 & 0,6 & 0,58 & 136137 & 0,94 & 0,9 \\
\hline 113785 & 0,89 & 0,85 & 136320 & 0,96 & 0,86 \\
\hline 116908 & 1 & 0,88 & 136456 & 0,62 & 0,5 \\
\hline 117005 & 0,96 & 0,88 & 136488 & 0,96 & 0,92 \\
\hline 117373 & 0,82 & 0,78 & 136571 & 0,98 & 0,94 \\
\hline 120425 & 0,92 & 0,84 & 137029 & 0,98 & 0,86 \\
\hline 121460 & 0,76 & 0,58 & 137435 & 0,74 & 0,74 \\
\hline 121886 & 0,96 & 0,88 & 137481 & 0,9 & 0,81 \\
\hline 123235 & 1 & 0,88 & 137701 & 0,78 & 0,68 \\
\hline 124015 & 0,96 & 0,9 & 138083 & 0,94 & 0,87 \\
\hline 124726 & 0,92 & 0,88 & 138642 & 0,85 & 0,85 \\
\hline
\end{tabular}

Legenda: ID: Número do prontuário; Pré: Índices pré-operatórios; Pós: Índices pósoperatórios.

Fonte: INTO/MS 2003-2007. 


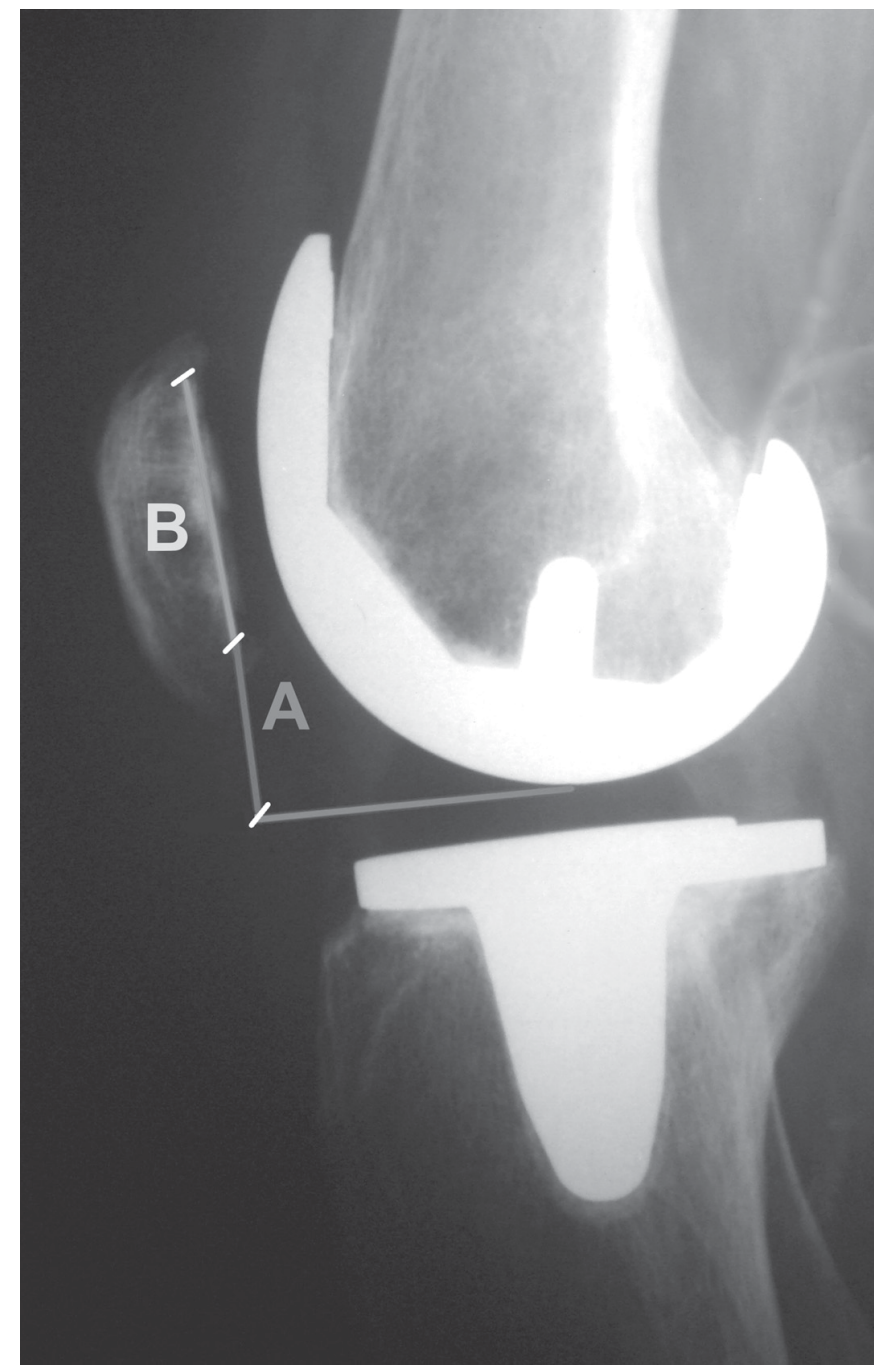

Figura 1 - Determinação do índice patelar pelo método de Blackburne-Peel, em radiografia na posição lateral, de artroplastia total do joelho

Para evitar discordâncias, todos os casos do estudo foram mensurados pelo mesmo observador. $\mathrm{Na}$ análise estatística foram avaliadas medidas de centro e dispersão e utilizado o teste $t$ de Student, na comparação entre grupos, com intervalo de confiança de $95 \%(\alpha=$ $5 \%$ ). O presente estudo foi submetido à avaliação e aprovado pela Comissão de Ética em Pesquisa do Instituto Nacional de Traumatologia e Ortopedia.

\section{RESULTADOS}

Os resultados obtidos foram analisados segundo parâmetros gráficos, descritivos e estatísticos.
O índice patelar pré-operatório médio do grupo I foi igual a 0,874 , sendo o menor igual a 0,60 e o maior, igual a 1 . No grupo II, o menor índice pré-operatório foi igual a 0,60 e o maior a 1 , sendo a média igual a 0,857 (tabela 3). Os índices médios de altura patelar, pós-operatórios dos grupos I e II estão expostos na tabela 4 e gráfico 1 .

\section{TABELA 3}

Medidas de centro e medida de dispersão pré-operatórias

\begin{tabular}{lcc}
\hline & $\begin{array}{c}\text { Grupo I } \\
\text { pré-operatório }\end{array}$ & $\begin{array}{c}\text { Grupo II } \\
\text { pré-operatório }\end{array}$ \\
\hline Média & 0,874 & 0,857 \\
Mediana & 0,88 & 0,88 \\
Moda & 0,96 & 0,96 \\
Desvio-padrão & 0,098 & 0,1 \\
\hline
\end{tabular}

Fonte: INTO/MS 2003-2007.

\section{TABELA 4}

Medidas de centro e medida de dispersão pós-operatórias

\begin{tabular}{lcc}
\hline & $\begin{array}{c}\text { Grupo I } \\
\text { pós-operatório }\end{array}$ & $\begin{array}{c}\text { Grupo II } \\
\text { pós-operatório }\end{array}$ \\
\hline Média & 0,849 & 0,787 \\
Mediana & 0,87 & 0,81 \\
Moda & 0,96 & 0,88 \\
Desvio-padrão & 0,104 & 0,113
\end{tabular}

Fonte: INTO/MS 2003-2007.

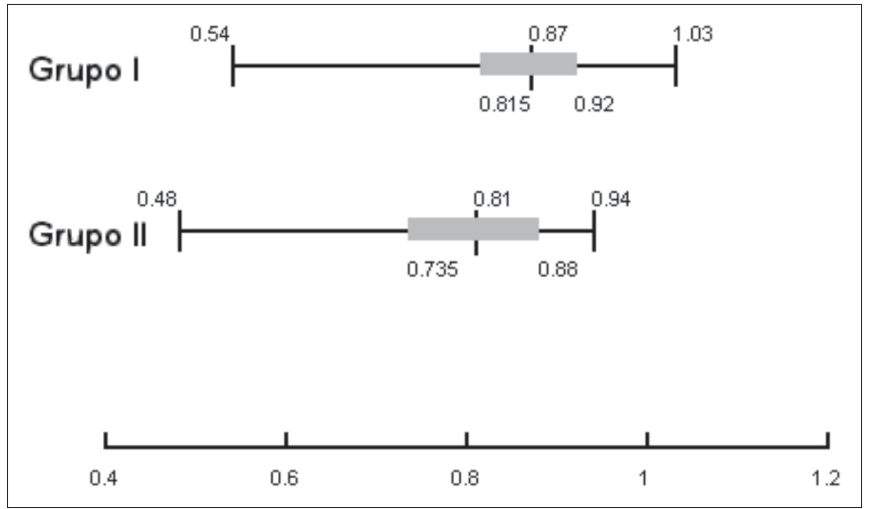

Gráfico 1 - Boxplot dos índices patelares dos grupos I e II Fonte: INTO/MS 2003-2007.

Foi observada tendência de rebaixamento da patela nos dois grupos. No grupo I o somatório dos índices patelares pré-operatórios foi igual a 52,48 e os índices 
pós-operatórios atingiram valores iguais a 50,96. No grupo II, o somatório dos valores pré-operatórios foi igual a 51,45, enquanto a soma dos índices pós-operatórios foi igual a 47,23.

Os dados da amostra fornecem evidências que permitem sustentar a existência de correlação linear entre as medidas pré e pós-operatórias de ambos os grupos, quanto ao desfecho do posicionamento patelar, de acordo com a análise gráfica e os coeficientes de correlação, dos grupos I $(r=0,86)$ e II $(r=0,88)$ (gráficos 2 e 3 ).

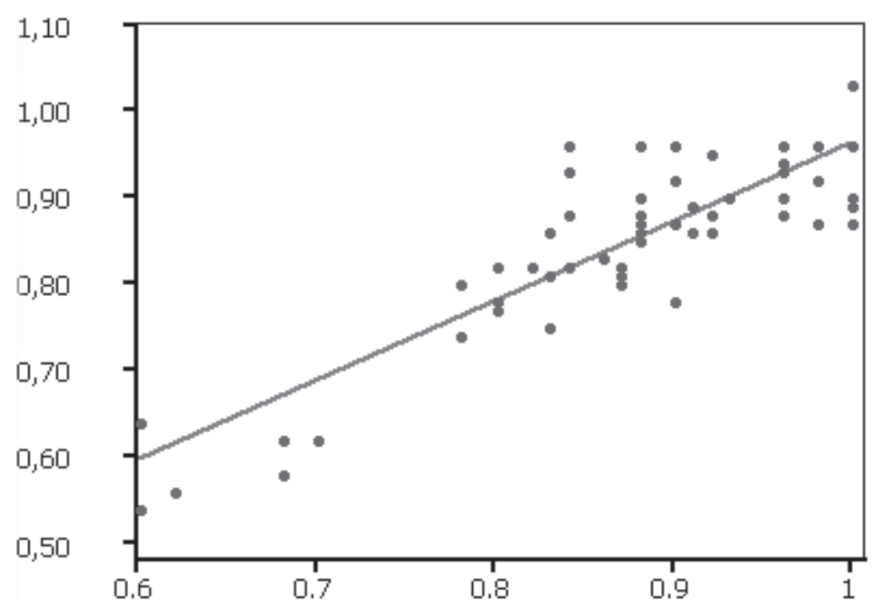

Gráfico 2 - Diagrama de dispersão com reta de regressão entre os índices pré e pós-operatórios do grupo I

Fonte: INTO/MS 2003-2007.

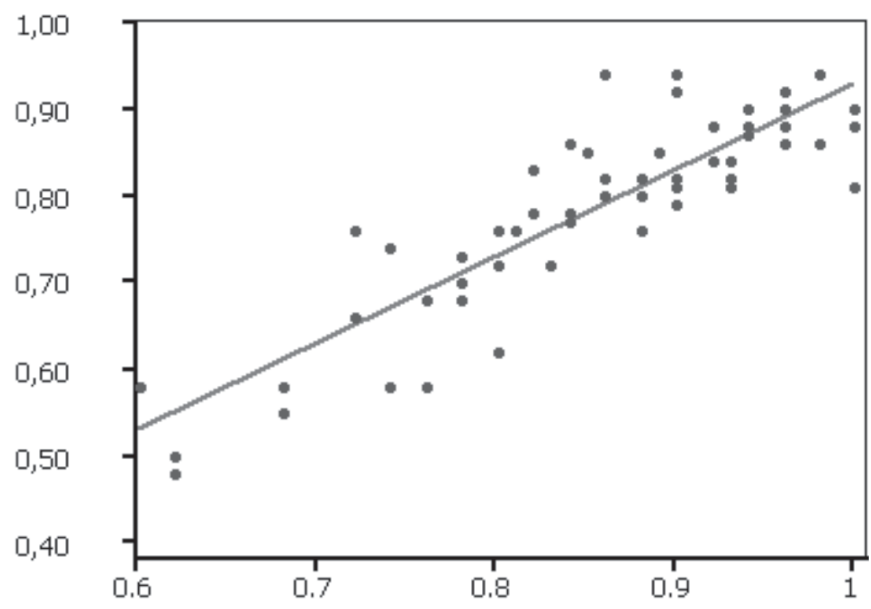

Gráfico 3 - Diagrama de dispersão com reta de regressão entre os índices pré e pós-operatórios do grupo II

Fonte: INTO/MS 2003-2007.
O teste $t$ de Student apresentou os seguintes valores, ao comparar os índices: pré-operatório $(\mathrm{p}=0,18808)$ e pós-operatório ( $\mathrm{p}=0,00068)$ dos grupos com e sem preservação do LCP. Assim, pode-se assumir a hipótese nula de igualdade entre os grupos I e II, no quesito índices patelares pré-operatórios ( $\mathrm{p}$-valor $>0,05$ ).

Entretanto, na questão valores pós-operatórios do índice patelar, rejeitou-se a hipótese nula de igualdade e assumiu-se a hipótese alternativa, ou seja, de desigualdade entre os grupos estudados: $\mathrm{p}<0,05$. Optou-se por essa forma de análise por não ser necessário apresentar concomitantemente o valor $t$ com o intervalo de confiança, bastando apenas conflitar o valor $\mathrm{p}$ com o nível de significância $\alpha=0,05(5 \%)$. Sendo o valor $\mathrm{p}$ menor que 0,05 , rejeita-se a hipótese nula e, caso contrário, aceita-se. A hipótese nula $\left(\mathrm{H}_{0}\right)$ evidencia que os grupos comparados são iguais e a hipótese alternativa $\left(\mathrm{H}_{1}\right)$ demonstra que houve diferença estatisticamente significativa entre os grupos.

No grupo I, na avaliação da altura patelar pós-artroplastia foram observados três casos de patela baixa $(5 \%)$ e um caso de patela alta $(1,66 \%)$, enquanto no grupo II houve sete casos de patela baixa $(11,66 \%)$ e nenhum de patela alta (gráfico 4).

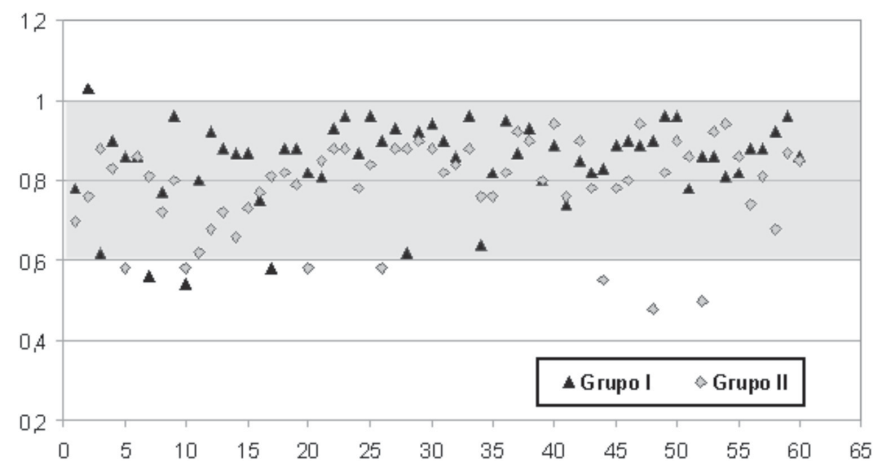

Gráfico 4 - Diagrama de dispersão dos índices pós-operatórios dos grupos I e II

Fonte: INTO/MS 2003-2007.

\section{DISCUSSÃO}

Para avaliação radiográfica da altura patelar existem diversos métodos descritos na literatura. Dentre eles os mais conhecidos: Insall-Salvati, BlackburnePeel, Caton-Deschamps e Blumensaat ${ }^{(5,16-18)}$. 
O índice escolhido para avaliação da altura patelar deste estudo foi o descrito por Blackburne-Peel. Nossa escolha resultou da associação de suas seguintes características: por ser amplamente utilizado na literatura ortopédica mundial pelo fato de ser do conhecimento da maioria dos ortopedistas e, principalmente, por se basear no estudo da acurácia de métodos radiológicos usados para se determinar a altura patelar ${ }^{(19)}$. O índice descrito por Insall et al apresenta acurácia semelhante à do método utilizado neste estudo; entretanto, a mensuração estaria mais sujeita a erros, devido, principalmente, à presença de osteófitos inferiores na patela e pela dificuldade, em algumas radiografias, de se visualizar o tubérculo tibial anterior ${ }^{(5)}$. A avaliação pós-operatória pelo método de Caton-Deschamps também pode ficar prejudicada pela presença do polietileno no planalto tibial e na superfície articular da patela $^{(17)}$. Pozzi et al, avaliaram a altura patelar pré e pós-artroplastia total do joelho pelos métodos de Caton-Deschamps e Blackburne-Peel, obtendo valores muito semelhantes ${ }^{(20)}$. Rogers et al compararam a reprodutibilidade interobservador de quatro métodos para avaliação do índice patelar após artroplastia total de joelho e concluíram que a reprodutibilidade da amostra foi maior quando foram avaliados os índices de Blackburne-Peel e Caton-Deschamps, que utilizam a linha articular protética, do que quando foram utilizados os índices de Insall-Salvati e Insall-Salvati modificado, que usam como referência a inserção do tendão patelar ${ }^{(21)}$.

Trabalhos que avaliaram próteses totais de joelho com problemas na relação patelofemoral concluíram que a realização da artroplastia em pacientes que apresentavam patela baixa pré-operatória, na maioria dos casos, estava relacionada com essa condição ${ }^{(13,21-23)}$. Para contornar esse possível fator de risco de desenvolvimento de patela baixa pós-artroplastia total de joelho, foram excluídos do nosso estudo todos os casos que apresentavam índice patelar fora do intervalo da normalidade no pré-operatório. A altura da interlinha articular pode ser alterada tanto pela espessura da ressecção proximal da tíbia e distal do fêmur, quanto pela espessura do polietileno utilizado. O flexo préoperatório do joelho, quando associado a grande re- tração das estruturas posteriores, é fator de risco para a ocorrência de patela baixa após artroplastia, devido à liberação de partes moles ou à realização de maior ressecção óssea que eventualmente é necessária nesses casos. Esses procedimentos podem resultar num alongamento relativo do aparelho extensor que, em última análise, leva à patela baixa dinâmica ${ }^{(21)}$. Aglietti et al afirmaram que o rebaixamento da patela deve sempre ser evitado, porém estimam que a elevação da altura da interlinha articular, devido ao uso de um platô tibial com polietileno mais espesso, é necessário para obter, em alguns casos, um joelho estável com as estruturas capsuloligamentares periféricas mais tensas ${ }^{(24)}$. Emodi et al relataram que pequenas alterações na posição da linha articular resultaram em significativas mudanças na tensão do LCP e na cinemática normal do joelho, e concluíram que todo esforço deve ser feito no sentido de restaurar a altura da linha articular ${ }^{(25)}$. Mihalko et al são de opinião de que, quanto maior a ressecção óssea tibial e maior a espessura do polietileno do platô tibial, mais baixa será a patela, porque a interlinha tende a ser mais alta, ou seja, é deslocada no sentido proximal ${ }^{(26)}$.

No nosso estudo não consideramos a espessura do polietileno utilizado, porque o objetivo primário foi avaliar a influência da preservação ou não do LCP na altura patelar. Entretanto, parece-nos bastante lógico que, quanto maior o espaço de extensão, dependente do nível de ressecção proximal da tíbia e do fêmur distal, maior a necessidade do aumento da espessura do polietileno, resultando na migração proximal da interlinha articular e conseqüente rebaixamento patelar. Uma medida que pode evitar essa ocorrência é a utilização de cunhas metálicas na extremidade distal do fêmur, possibilitando o uso de componentes de polietileno de menor espessura, desde que compatível com o espaço de flexão. Outro fator que pode influenciar na posição da patela em relação à interlinha articular é a opção de se preservar ou não o LCP nas artroplastias totais de joelho. Dessa forma, segundo certos autores, o sistema que não conserva o LCP poderia, eventualmente, trazer problemas de rebaixamento da patela, devido à migração proximal da interlinha articular pelo aumento do espaço de flexão(4,14). 
Mihalko et al concluíram que o principal resultado da ressecção do LCP é o aumento do espaço de flexão e que este fato pressupõe mudanças na altura da linha $\operatorname{articular}^{(26)}$. Kadoya et al avaliaram o efeito da ressecção do LCP em 30 pacientes submetidos à artroplastia total de joelho e também concluíram que o principal efeito da ressecção do LCP foi o aumento do espaço de flexão e, portanto, este espaço poderia ser controlado seletivamente com a liberação do $\mathrm{LCP}^{(27)}$. Pozzi et al avaliaram a altura patelar pré e pós-operatória de 161 joelhos submetidos à artroplastia total utilizando cinco diferentes modelos de prótese, sendo que somente um deles não conservava o LCP. Nesse estudo foram encontrados oito casos de patela baixa e cinco de patela alta no pré-operatório. No pós-operatório, o número de patelas baixas aumentou para 19 e de patelas altas diminuiu para dois casos. Os autores observaram que os modelos de próteses que conservavam o LCP apresentaram médias de índices da altura patelar inferiores àquelas do modelo que o sacrificavam. Nos pacientes que apresentavam patela baixa pré-operatória, houve persistência da patela baixa no pós-operatório, ou até agravamento do problema ${ }^{(20)}$. Koshino et al avaliaram o índice patelar pelo método de Insall-Salvati em 94 joelhos e observaram diminuição de pelo menos $10 \%$ do índice em 61 joelhos após artroplastia total; em 31 casos o índice não foi alterado e em somente dois casos houve aumento do índice patelar ${ }^{(22)}$. Nos estudos acima citados, um viés a considerar é a não inclusão de casos que pré-operatoriamente já se apresentavam com o índice patelar abaixo do limite da normalidade.

Entre os pacientes que avaliamos, encontramos diminuição da altura patelar em ambos os grupos submetidos à artroplastia total de joelho. Porém, essa foi mais evidente no grupo em que houve sacrifício do LCP, quando comparado com o grupo que utilizou implantes com preservação desse ligamento $(11,6 \%$ e $5 \%$ respectivamente).

Kubota et al, após avaliar a articulação patelofemoral de 35 joelhos submetidos à artroplastia total de joelho preservando o LCP, encontraram seis joelhos $(17,14 \%)$ com índice patelar de Blackburne et al abaixo do normal $(0,60)^{(23)}$. Em nosso estudo, o grupo em que se preservou o LCP apresentou um percentual menor de patelas baixas (5\% - três casos), porém, devemos lembrar que nele foram excluídos todos os casos de patela baixa pré-operatórios, o que não aconteceu no estudo de Kubota et $a l^{(23)}$.

A ocorrência de patela alta, por outro lado, não é um achado usual após artroplastia total de joelho. Geralmente, está relacionada à ruptura pós-operatória do ligamento patelar ou ao rebaixamento da linha articular. Observamos apenas um caso com patela alta pósoperatória, no grupo em que se preservou o LCP $(1,66 \%)$. Cabe ressaltar que esse joelho já apresentava, pré-operatoriamente, índice patelar no seu limite superior (índice $=1)$.

\section{CONCLUSÕES}

Foi observada tendência à diminuição da altura patelar em ambos os grupos estudados, sendo esta mais evidente no grupo em que houve sacrifício do LCP.

\section{REFERÊNCIAS}

1. Krevolin JL, Pandy MG, Pearce JC. Moment arm of the patellar tendon in the human knee. J Biomech. 2004;37(5):785-8.

2. Wahrenberg H, Lindbeck L, Ekholm J. Knee muscular moment, tendon tension force and EMG during a vigorous movement in man. Scand J Rehabil Med. 1978;10(2):99-106.

3. Grana WA, Kriegshauser LA. Scientific basis of extensor mechanism disorders. Clin Sports Med. 1985;4(2):247-57.

4. Grelsamer RP. Patella baja after total knee arthroplasty: is it really patella baja? J Arthroplasty. 2002;17(1):66-9.

5. Insall J, Salvati E. Patella position in the normal knee joint. Radiology. 1971;101(1):101-4.

6. Aparicio G, Abril JC, Calvo E, Alvarez L. Radiologic study of patellar height in Osgood-Schlatter disease. J Pediatr Orthop. 1997;17(1):63-6.

7. Bryan RS, Rand JA. Revision total knee arthroplasty. Clin Orthop Relat Res. 1982;(170):116-22.

8. Cameron HU, Hunter GA. Failure in total knee arthroplasty: mechanisms, revisions, and results. Clin Orthop Relat Res. 1982;(170):141-6.

9. Clayton ML, Thirupathi R. Patellar complications after total condylar arthroplasty. Clin Orthop Relat Res. 1982;(170):152-5.

10. Mochizuki RM, Schurman DJ. Patellar complications following total knee arthroplasty. J Bone Joint Surg Am. 1979; 61(6A):879-83.

11. Insall JN, Lachiewicz PF, Burstein AH. The posterior stabilized condylar prosthesis: a modification of the total condylar 
design. Two to four-year clinical experience. J Bone Joint Surg Am. 1982;64(9):1317-23.

12. Vinciguerra B, Pascarel X, Honton JL. [Results of total knee prostheses with or without preservation of the posterior cruciate ligament]. Rev Chir Orthop Reparatrice Appar Mot. 1994;80(7):620-5. French.

13. Figgie HE 3rd, Goldberg VM, Heiple KG, Moller HS 3rd, Gordon NH. The influence of tibial-patellofemoral location on function of knee in patients with the posterior stabilized condylar knee prosthesis. J Bone Joint Surg Am. 1986;68(7): 1035-40.

14. Dejour D, Levigne C, Dejour H. [Postoperative low patella. Treatment by lengthening of the patellar tendon]. Rev Chir Orthop Reparatrice Appar Mot. 1995;81(4):286-95. French.

15. Kim W, Rand JA, Chao EY. Biomechanics of the knee. In: Rand JA, editor. Total knee arthroplasty. New York: Raven Press; 1993. p. 9-57.

16. Blackburne JS, Peel TE. A new method of measuring patellar height. J Bone Joint Surg Br. 1977;59(2):241-2.

17. Caton J, Deschamps G, Chambat P, Lerat JL, Dejour H. [Patella infera. Apropos de 128 cases]. Rev Chir Orthop Reparatrice Appar Mot. 1982;68(5):317-25.

18. Blumensaat C. [Die Lageabweichungen und Verrenkungen der Kniescheibe]. Ergeb Chir Orthop. 1938;31:149 -223. German.

19. Berg EE, Mason SL, Lucas MJ. Patellar height ratios. A comparison of four measurement methods. Am J Sports Med. 1996;24(2):218-21.
20. Pozzi JF, Konkewicz ER, Nora B, Rodrigues L. A altura da patela em próteses totais de joelho. Rev Bras Ortop. 1997; 32(5):367-73.

21. Rogers BA, Thornton-Bott P, Cannon SR, Briggs TW. Interobserver variation in the measurement of patellar height after total knee arthroplasty. J Bone Joint Surg Br. 2006;88(4): 484-8.

22. Koshino T, Ejima M, Okamoto R, Morii T. Gradual low riding of the patella during postoperative course after total knee arthroplasty in osteoarthritis and rheumatoid arthritis. J Arthroplasty. 1990;5(4):323-7.

23. Kubota MS, Navarro RD, Carneiro Filho M, Luzo MVM. Estudo da articulação patelofemoral na artroplastia total do joelho cimentada que preserva o ligamento cruzado posterior. Rev Bras Ortop. 2004;9(1/2):21-9.

24. Aglietti P, Rinonapoli E. Total condylar knee arthroplasty. A five-year follow-up study of 33 knees. Clin Orthop Relat Res. 1984;(186):104-11.

25. Emodi GJ, Callaghan JJ, Pedersen DR, Brown TD. Posterior cruciate ligament function following total knee arthroplasty: the effect of joint line elevation. Iowa Orthop J. 1999;19:82-92.

26. Mihalko WM, Krackow KA. Posterior cruciate ligament effects on the flexion space in total knee arthroplasty. Clin Orthop Relat Res. 1999;(360):243-50.

27. Kadoya Y, Kobayashi A, Komatsu T, Nakagawa S, Yamano Y. Effects of posterior cruciate ligament resection on the tibiofemoral joint gap. Clin Orthop Relat Res. 2001;(391):210-7. 